\title{
La calidad del servicio en el sistema de transporte público y su impacto en la satisfacción del usuario (Quality service in the public transportation system and its impact in user's satisfaction)
}

\author{
' Luis Enrique Ibarra-Morales \\ iNatalia Guadalupe Romero-Vivar \\ iiiDaniel Paredes-Zempual
}

\begin{abstract}
Giving an excellent service trough quality attributes has been the constant in most organizations, now that it has a direct impact in client's satisfaction as a user of the services, and in the strategic results of the organization. This research study makes a contribution to the area of administration and business, as it measures the quality service and clients' satisfaction of the public transportation system in Hermosillo, Sonora, México. This investigation is a quantitative type with a universe of study of 1296 transport users, to whom was applied a measurement instrument where were determined the dimensions or attributes with the highest impact in client's satisfaction, through the Servperf (Service Performance) methodology. The analysis was carried out through an exploratory factor analysis (EFA), as well as a multiple linear regression technique. The dimensions evaluated were: Tangible, reliability, responsibility, assurance and empathy. The results of the estimation of multiple linear regression determine the importance, weight and value of each dimension in terms of satisfaction measured by the quality service given and for this study, indicate the areas that have to be attended and improved for the clients to be more satisfied with the service in terms of quality.
\end{abstract}

Key words: quality service, public transportation system, servperf, user's satisfaction.

JEL: L25, L91, M31.

Resumen. El brindar un excelente servicio a través del atributo de la calidad, ha sido la constante en la mayoría de las empresas, ya que tiene un impacto directo en la satisfacción del cliente como usuario de los servicios y, en los resultados estratégicos de la organización. La presente investigación realiza un aporte al área de la administración y negocios, ya que mide la calidad del servicio y la satisfacción del usuario del sistema de transporte público en Hermosillo, Sonora, México. Esta investigación es de tipo cuantitativa con un universo de estudio de 1,296 usuarios del transporte, a los cuales se les aplicó un instrumento de medición donde se determinaron las dimensiones o atributos que más impactan en la satisfacción del cliente haciendo uso de la

\footnotetext{
' UES. Ley Federal del Trabajo s/n, Hermosillo, Sonora. Email: luisim00@hotmail.com

"i UES. Ley Federal del Trabajo s/n, Hermosillo, Sonora.

iii UES. Ley Federal del Trabajo s/n, Hermosillo, Sonora.
} 
metodología Servperf (Service Performance). El análisis se llevó a cabo a través de un análisis factorial exploratorio (AFE); así como la técnica de regresión lineal múltiple. Las dimensiones evaluadas fueron: Tangibles, confiabilidad, responsabilidad, seguridad y, empatía. Los resultados de la estimación de regresión lineal múltiple determinan la importancia, peso y valor de cada dimensión en términos de satisfacción medido por la calidad del servicio otorgado y que, para este estudio, indican las áreas que se deben de atender y mejorar para que el usuario se sienta más satisfecho con el servicio en términos de calidad.

Palabras clave: calidad del servicio, satisfacción del usuario, servperf, sistema de transporte público.

\section{Introducción}

El crecimiento demográfico acelerado en la capital del estado de Sonora, ha propiciado el incremento en el uso del transporte público como un medio para desplazarse entre los diferentes puntos de interés, lo que ha generado a su vez, una serie de problemas colaterales como: sociales, medioambientales, de tránsito vehicular, económicos, políticos y logísticos, impactando significativamente la calidad de vida de los usuarios y habitantes de la ciudad.

El sistema de transporte público en la capital del estado de Sonora, ha sido por años un problema que parece no tener fin, ya que adolece de un plan maestro de crecimiento ordenado y de atención integral al usuario que, por un lado, disminuyan los problemas e impactos sociales en materia de movilización de usuarios, logística de operación de unidades y rutas (tiempos de llegada como de partida, eficiencia y eficacia en el manejo de camiones) y, por otro lado, que permita operar un sistema de transporte público moderno que contravenga al sistema actual y minimice los altos impactos en asuntos de contaminación y deterioro del medio ambiente, así como la calidad de vida de los que hacen uso de él diariamente.

En ese sentido, se necesita desarrollar un sistema de transporte público que atienda a las necesidades presentes y futuras de los usuarios que, a diario tienen que enfrentar las malas costumbres y culturas ya adoptadas por los chóferes de transporte. Aspectos como adoptar y transitar hacia una nueva cultura entre los ciudadanos y usuarios, los líderes del sistema de transporte público y los funcionarios del gobierno que, en su conjunto, incite a una mejor toma de decisiones centradas en la calidad del servicio y en una mayor satisfacción de los usuarios. 
La estructura del presente artículo está basada en el método científico, identificando el planteamiento del problema, el objetivo de la investigación, la hipótesis de investigación, el marco teórico, la metodología utilizada, el instrumento de medición, diseño, presentación y discusión de resultados.

\section{Planteamiento del problema}

El medir la calidad en el servicio y la satisfacción del usuario, se hace cada vez más necesario para las empresas, y para el sector del transporte público no es la excepción, sobre todo cuando las percepciones de los clientes cada vez son más exigentes. Phau y Ferguson (2013), señalan que la satisfacción del cliente está intrínsecamente relacionada con la calidad percibida en el servicio, pese a que ambos constructos son a menudo confundidos. En efecto, diferentes autores como Bhardwaj (2011) o Khan, Majid, \& Yaqoob (2013), han manifestado que las dimensiones utilizadas para evaluar la calidad en el servicio influyen positivamente en la satisfacción del cliente.

Con base a la problemática presentada y a la propia necesidad de medir la percepción de los usuarios del transporte público, surge la siguiente pregunta de investigación, ¿cuáles son las dimensiones de la calidad en el servicio que se relacionan de forma positiva con la satisfacción del usuario?

\section{Objetivo general de la investigación}

Cabe destacar que el objetivo principal de UNE Transporte Urbano de Sonora es mejorar el servicio de atención al usuario a través de la profesionalización de los servicios, certificación de chóferes, paradas modernas y confortables, más y mejores autobuses con horarios confiables y menos tiempo de espera. En coherencia con la UNE, el objetivo general de este artículo es medir la calidad del servicio mediante las cinco dimensiones utilizadas en la metodología Servperf desarrollada por Cronin y Taylor (1992, 1994), y su impacto e influencia en la satisfacción del usuario del sistema de transporte público.

\section{Hipótesis de investigación}

Para ello, se propone analizar las siguientes hipótesis a partir de las dimensiones para medir la calidad del servicio: Tangibles (apariencia de las unidades de trans- 
porte, personal y equipo), confiabilidad (habilidad para ejecutar el servicio prometido de forma fiable y cuidadosa), responsabilidad (disposición y voluntad para ayudar al usuario y proporcionar un servicio rápido y confiable), seguridad (conocimiento, atención y habilidades mostradas para inspirar credibilidad y confianza) y, empatía (atención individualizada que se ofrece en el servicio que se brinda).

$\mathrm{H}_{1}$ : Existe una relación positiva entre la dimensión tangibles para evaluar la calidad en el servicio y la satisfacción del usuario.

$\mathrm{H}_{2}$ : Existe una relación positiva entre la dimensión confiabilidad para evaluar la calidad en el servicio y la satisfacción del usuario.

$\mathrm{H}_{3}$ : Existe una relación positiva entre la dimensión responsabilidad para evaluar la calidad en el servicio y la satisfacción del usuario.

$\mathrm{H}_{4}$ : Existe una relación positiva entre la dimensión seguridad para evaluar la calidad en el servicio y la satisfacción del usuario.

$\mathrm{H}_{5}$ : Existe una relación positiva entre la dimensión empatía para evaluar la calidad en el servicio y la satisfacción del usuario.

\section{Marco teórico}

\section{Servicio al cliente}

El servicio al cliente tal y como la describe Malcom Peel (1993, p. 24) citado por Frometa Vázquez, Zayas Ramos y Pérez Martínez (2008), es definida como aquella actividad que relaciona la empresa con el cliente a fin de que éste quede satisfecho con dicha actividad. Servicio al cliente es el establecimiento y la gestión de una relación de mutua satisfacción de expectativas entre el cliente y la organización. Para ello, se vale de la interacción y retroalimentación entre personas, en todas las etapas del proceso del servicio (Lovelock, 1990, p. 491). El objetivo básico es mejorar las experiencias que el cliente tiene con el servicio de la organización (Lovelock, 1990).

Muchos clientes, no solo desean un servicio mejor, sino que lo esperan (Denton, 1991). Hay dos actitudes básicas del servicio al cliente, la actitud positiva (que hace referencia a un excelente comportamiento ante el cliente) y la actitud negativa (mal comportamiento ante el cliente), aun cuando en las políticas de actuación de la organización esté consignado su interés y su propósito de brindar un excelente servicio que permita fidelizar a los clientes y desarrollar así una ventaja competitiva. 


\section{Calidad en el servicio}

Son muchos los autores que de una u otra forma han abordado el concepto de la calidad. Algunas de ellas se han definido como "el resultado de comparación de las expectativas y percepciones" según Zeithaml (1988) en Vásquez (2010); Juran y Gryna (1993), se refieren a la calidad como el conjunto de características que satisfacen las necesidades del consumidor.

Por lo tanto, la calidad del servicio y satisfacción del usuario son conceptos que están íntimamente relacionados puesto que una mayor calidad del servicio llevará a aumentar la satisfacción del consumidor, es decir, que la primera debe ser tratada como un antecedente de la satisfacción (Parasuraman, Zeithaml y Berry, 1985).

En la literatura sobre la calidad del servicio, el concepto de calidad se refiere a la calidad percibida, es decir "al juicio del consumidor sobre la excelencia y superioridad de un producto" (Zeithaml, 1988, p. 3). En términos de servicio significaría "un juicio global, o actitud, relacionada con la superioridad del servicio" (Parasuraman, Zeithaml y Berry, 1988, p. 16).

Grönroos (2001), reconoce que la línea que separa las evaluaciones de la calidad de las otras tendencias de evaluación de experiencias de servicio no está muy bien definida. A diferencia de la calidad que se mide en los productos en términos de defectos o durabilidad de los mismos, la calidad de los servicios se torna complicado de medir (Parasuraman et al., 1988). La intangibilidad de los servicios origina que éstos sean percibidos en gran medida de una forma subjetiva por los consumidores o clientes (Grönroos, 1994, p. 37).

\section{Satisfacción del consumidor}

Según los autores Zeithaml \& Parasuraman (2004), afirman que, para medir la satisfacción del cliente con varios aspectos de la calidad del servicio, se basa en la premisa de que los clientes pueden evaluar la calidad del servicio de una empresa al comparar las percepciones que tienen del servicio con sus propias expectativas.

En un sentido amplio, la satisfacción, según numerosos autores es el cumplimiento de las expectativas del usuario. Es un concepto psicológico, implica el sentimiento de bienestar y placer por obtener lo que se desea y espera de un producto o servicio (Sancho, 1998). Por ello, los niveles de satisfacción del usuario 
es una forma directa de medir la complacencia de las expectativas, necesidades y deseos de los usuarios. De lo anterior, se deduce la estrecha relación entre la calidad del servicio y la satisfacción del cliente (Morillo, M., y Morillo, M. Del C., 2016).

Para autores como Münch (1998), existe cierto orden e indica que la satisfacción precede a la calidad del servicio, y que la calidad se alcanza con la "... satisfacción total de las necesidades del cliente, mediante la prestación de actividades esencialmente intangibles con un valor agregado y el cumplimiento de los requisitos adecuados al producto o servicio" (p. 179). Según Kotler y Armstrong (2013), la calidad se vincula con el valor y la satisfacción del cliente.

Hoffman y Bateson (2012), indican que, "...la satisfacción de los clientes es una medida de corto plazo, específica de la transacción, mientras que la calidad de los servicios es una actitud a largo plazo debida a la evaluación global de un desempeño" (p. 324). Adicionalmente para Lovelock et al. (2010), la satisfacción es un concepto dinámico que evoluciona con el paso del tiempo y que no se debe interpretar sólo desde la perspectiva de una sola transacción, en otras oportunidades hasta la satisfacción e insatisfacción de otros miembros de la familia o vínculos sociales del individuo contribuyen a la formación de la calidad del servicio.

Por lo antes mencionado, Phau y Ferguson (2013), señalan que la satisfacción del cliente está intrínsecamente relacionada con la calidad percibida en el servicio, pese a que ambos constructos son a menudo confundidos. En efecto, diferentes autores como Bhardwaj (2011) o Khan et al. (2013), han destacado que los atributos usados para evaluar la calidad en el servicio influyen positivamente en la satisfacción del cliente.

\section{Service Performance (Servperf)}

Los autores Cronin y Taylor (1992), establecieron una escala más concisa que Servqual y la llamaron Servperf. Esta nueva escala está basada exclusivamente en la valoración de las percepciones, con una evaluación similar a la escala Servqual, la cual tiene en cuenta tanto las expectativas como las percepciones. Continuando con Cronin y Taylor (1992), se basan en Carman (1990), para afirmar que la escala Servqual (teoría de gaps de Parasuraman, Zeithaml y Berry, [1985]) no presentaba mucho apoyo teórico y evidencia empírica como punto de partida para medir la calidad de servicio percibida. 
Las diez dimensiones iniciales que desarrollaron Parasuraman, Zeithaml y Berry (1988, p. 26), después de varios estudios estadísticos permitieron encontrar ciertas correlaciones y reducir a cinco el número de dimensiones que determinan la calidad del servicio, siendo éstas las que actualmente son utilizadas por la metodología Servperf de Cronin y Taylor (1992).

Las cinco dimensiones de la escala usada por Servperf son: Confianza o empatía: muestra de interés y nivel de atención individualizada que ofrecen las empresas a sus clientes; Fiabilidad: habilidad para ejecutar el servicio prometido de forma fiable y cuidadosa; Responsabilidad: seguridad, conocimiento y atención de los empleados y su habilidad para inspirar credibilidad y confianza; Capacidad de respuesta: disposición para ayudar a los clientes y para prestarles un servicio rápido y; Tangibilidad: apariencia de las instalaciones físicas, equipos, personal y materiales de comunicación.

\section{El servicio de transporte público}

Hoy en día, con el crecimiento demográfico y la globalización, la concentración de población en los asentamientos urbanos se ha ido incrementando con la movilización de personas que migran buscando calidad de vida, mejores condiciones de vivienda, así como también por trabajo. Esto ha traído como consecuencia que la utilización del transporte urbano se convierta en una estrategia para la movilización diaria; el sistema de conexión terrestre dentro de la vialidad urbana colapsa con la cantidad de automóviles que diariamente transitan por la ciudad. Por lo que el servicio de transporte público representa una oportunidad para movilizarse sin necesidad de utilizar los vehículos propios; sin embargo, en la ciudad de Hermosillo, Sonora, se han detectado distintas situaciones en el servicio y la calidad del mismo.

Delgado Martínez et al., (2011) cita a Molinero y Sánchez (1997), donde comenta que la calidad en el sistema de transporte público es un concepto amplio que engloba varios aspectos, entre los que se incluyen consideraciones relativas a la comodidad y seguridad dentro del vehículo, los tiempos empleados en la realización del trayecto, y la conveniencia y existencia de infraestructura que apoye al servicio.

Navarrete y Andrade (2010), señalan a Barry (2004), Hovell (1999), Disney (1998), como autores internacionales que abordan variables de eficiencia en el transporte, dichas aportaciones concluyen que los factores comodidad, limpieza 
y rapidez en el transporte público son importantes para el desarrollo y el buen servicio, también deducen que los automovilistas no utilizarían su automóvil para liberar las vialidades, en caso de que el transporte público garantizara su comodidad, eficiencia y reducción de tiempo con infraestructuras modernas y operativas, en ese sentido, la calidad en el transporte urbano se convierte en un factor decisivo para incrementar su uso.

La descripción de la oferta de transporte público municipal incluye características como: la cantidad de rutas, horarios de servicio, frecuencia e intervalos de llegadas, estado físico de las unidades, cortesía en el trato al usuario y manejo eficiente del transporte agrupadas todos ellas en cinco dimensiones para evaluar la calidad del servicio.

De acuerdo a la literatura existente, se distinguen tres enfoques para identificar los factores que definen la calidad del servicio en el transporte público. El primero se basa en mediciones del desempeño del nivel de servicio (Sussman, 2000). Tal desempeño se determina desde la perspectiva de los conductores, reguladores y usuarios.

El segundo enfoque está basado en la valoración por medio de encuestas de satisfacción del usuario y la detección de áreas de oportunidad entre los servicios ofertados y deseados. Finalmente, el tercer enfoque utiliza la teoría microeconómica del consumidor para estimar, a partir de la especificación de un modelo de elección discreta (Ben-Akiva \& Lerman, 1985; Ortúzar, 2000), una función de utilidad integrada por los factores que definen la calidad del servicio (Rizzi y Ortúzar, 2003; Hojman, Ortúzar, Rizzi, 2003).

\section{Metodología}

En cuanto a la metodología de la investigación realizada, fue de tipo exploratoria, descriptiva y correlacional, ya que fue necesario describir y comprobar qué relación existe entre la calidad del servicio y la satisfacción del usuario del transporte público municipal.

La metodología cuantitativa consistió en la recolección de la información, para lo cual se aplicaron 1,296 encuestas a sujetos seleccionados al azar, y que sirvieron para medir las percepciones de los usuarios en las 31 rutas del servicio de transporte público urbano de Hermosillo, Sonora. La recolección de información tuvo lugar en Hermosillo, Sonora, y se realizó en los meses de noviembre a diciembre del año 2016. 
La construcción del marco teórico se hizo a través de la revisión bibliográfica y la contextualización de los cuestionarios. La revisión bibliográfica consistió en estudiar las teorías, los conceptos, las dimensiones y los instrumentos para evaluar la calidad del servicio, propuesta por Parasuraman et al., (1985) y adaptada al transporte público. Se diseñó el borrador del instrumento únicamente para las percepciones del usuario.

Después se aplicó el instrumento de medición a una muestra piloto de 30 usuarios y, posteriormente, se ajustó al diseño final del instrumento quedando integradas las cinco dimensiones que miden la calidad en el servicio en 25 ítems distribuidos equitativamente como sigue: DIM1: Tangibles; DIM2: Confiabilidad; DIM3: Responsabilidad; DIM4: Seguridad y; DIM5: Empatía.

\section{Universo y marco muestral}

La población que se analizó para valorar y probar cuantitativamente la calidad del servicio sobre la satisfacción del usuario del sistema de transporte público municipal, fue de 150,000 usuarios en la ciudad de Hermosillo, Sonora. Se utilizó una muestra aleatoria de 1,296 usuarios del transporte público municipal. El nivel de confianza utilizado para asegurar la confiabilidad de los datos fue del $97 \%$, con un margen de error del $3 \%$ y un nivel de heterogeneidad del $50 \%$.

\section{Instrumento de medición}

Para recoger la información se utilizó un cuestionario con 34 preguntas divididas en dos grandes bloques. Bloque I, con 8 preguntas para conocer el perfil del encuestado y sus preferencias en las rutas y, el Bloque II, con 26 preguntas para evaluar las cinco dimensiones de la calidad en el servicio. La escala utilizada fue Likert de cinco puntuaciones que van desde 1) Totalmente en desacuerdo hasta 5) Totalmente de acuerdo. Para el análisis de datos en esta investigación se utilizó un análisis factorial y la técnica de regresión lineal múltiple para evaluar el ajuste del modelo propuesto en aras de medir la satisfacción del usuario con el servicio. 


\section{Presentación de resultados}

\section{Análisis de fiabilidad y consistencia interna}

Para la prueba piloto se procedió con la aplicación del instrumento de medición a 30 sujetos con la intención de realizar la prueba estadística necesaria para validar los constructos. En resumen, se realizó un análisis a través del alfa de Cronbach para cada uno de los 25 ítems que conforman las dimensiones de la calidad en el servicio. Este análisis permitió estimar la fiabilidad del instrumento de medida y asegurar que midieran el mismo constructo o dimensión teórica. En ese sentido, fue importante establecer la consistencia de los ítems considerados para cada una de las variables o dimensiones, con el fin de ser utilizados como referencia al generar un modelo de regresión lineal múltiple.

Como criterio general, George y Mallery (2003, p. 231), sugieren las recomendaciones siguientes para evaluar los coeficientes de alfa de Cronbach: $>0.9$ es excelente; $>0.8$ es bueno; $>0.7$ es aceptable; $>0.6$ es cuestionable; $>0.5$ es pobre $y_{;}<0.5$ es inaceptable. En la Tabla 1, se muestran las correlaciones inter-ítem por cada dimensión donde se puede apreciar que el intervalo oscila entre 0.600 y 0.396 , valores que son considerados buenos (Merino, Navarro y García, 2014). Asimismo, se aprecia una alfa de Cronbach satisfactoria para las cinco dimensiones. Por lo tanto, no hubo necesidad de eliminar algún ítem del cuestionario.

Tabla 1. Correlación entre dimensiones y alfa de Cronbach de las dimensiones

\begin{tabular}{cccc}
\hline Dimensión & No de ítems & rii & Alfa de Cronbach (a) \\
\hline DIM1 & 5 & 0.502 & 0.834 \\
DIM2 & 5 & 0.396 & 0.766 \\
DIM3 & 5 & 0.570 & 0.869 \\
DIM4 & 5 & 0.577 & 0.872 \\
DIM5 & 5 & 0.600 & 0.882 \\
\hline
\end{tabular}

Nota: rii: correlación inter-ítem promedio. Fuente: Elaboración propia, a partir de los datos procesados en el SPSS, v23

\section{Prueba de adecuación muestral}

La medida de adecuación muestral a través de la prueba Kaiser-Meyer-Olkin, implica que la relación entre las variables es alta (KMO $\geq 0.9$ ). La prueba de esfericidad de Bartlett evalúa la aplicabilidad del análisis factorial de las variables estudiadas. El modelo fue significativo, ya que $(p<0.05)$. El análisis factorial es factible de llevarse a cabo, tal y como se muestra en la Tabla 2. 
Tabla 2. KMO y Prueba de Bartlett para las variables

\begin{tabular}{ccc}
\hline \multicolumn{2}{c}{ Prueba } & Valor \\
\hline Medida de adecuación muestral de Kaiser-Meyer-Olkin. & .966 \\
& Chi-cuadrado aproximado & 19635.986 \\
Prueba de esfericidad de & gl & 300 \\
Bartlett & Sig. & .000 \\
\hline
\end{tabular}

Fuente: Elaboración propia, a partir de los datos procesados en el SPSS, v23

\section{Prueba de unidimensionalidad}

Esta prueba implica que un sólo rasgo latente o constructo se encuentra en la base de un conjunto de ítems (Hattie, 1985). Lo importante es que la mayor cantidad de la varianza observada en las respuestas a los ítems sea explicada por un solo atributo latente (Embretson \& Reise, 2000). Así, un conjunto de ítems será unidimensional si el primer factor explica por lo menos el $40 \%$ de la varianza total (Carmines \& Zeller, 1979), tal y como se indica en la Tabla 3, donde la varianza total explicada para cada una de las dimensiones, cumple con el supuesto de unidimensionalidad de la escala según lo propuesto por Carmines \& Zeller (1979).

Tabla 3. Varianza total explicada por cada dimensión

\begin{tabular}{ccc}
\hline Dimensión & \multicolumn{2}{c}{$\begin{array}{c}\text { Varianza total explicada } \\
\text { \% de varianza }\end{array}$} \\
\hline DIM1: Tangibles & 3.033 & 60.657 \\
DIM2: Confiabilidad & 2.651 & 53.026 \\
DIM3: Responsabilidad & 3.297 & 65.941 \\
DIM4: Seguridad & 3.311 & 66.225 \\
DIM5: Empatía & 3.402 & 68.036 \\
\hline
\end{tabular}

Fuente: Elaboración propia, a partir de los datos procesados en el SPSS, v23

\section{Análisis factorial}

Se realizó un análisis factorial, a través del método de extracción de análisis de componentes principales y el método de rotación VARIMAX, con la finalidad de hallar aquellos componentes o factores que se caracterizan por estar ínter-correlacionados entre sí y, que sucesivamente expliquen la mayor parte de la varianza total. En la Tabla 4, se aprecia que los 25 ítems cargan en tres dimensiones. 


Ítem
22. El conductor muestra siempre interés en solucionar cualquier dificultad que se presenta durante el servicio
o traslado.
17. El conductor mostró e inspiró confianza en el servicio y en la atención que brindó.
25. El conductor siempre es respetuoso y prudente al momento de manejar la unidad de transporte público
colectivo.
24. El conductor le brinda una atención personalizada, antes, durante y después del servicio que otorga.
21. El conductor lo atiende y lo trata con amabilidad, respeto y paciencia.
20. El viajar en autobús me da la confianza y seguridad que llegaré a tiempo a mi destino final.
19. Los problemas o dificultades que se presentan durante el servicio y atención se resuelven en tiempo y
mejoran notablemente.

16. El conductor le brindó el tiempo suficiente para responder a todas las dudas o preguntas sobre el servicio.

18. El conductor mostró sus habilidades y destrezas en el manejo, respetando siempre las reglas y señalamientos de tránsito.

23. El autobús ofrece otros servicios como aire acondicionado, rampas para subir y bajar de la unidad, entre otras comodidades.

14. La atención que se brinda por parte del personal encargado de la unidad de transporte, es rápido, confiable y seguro.

2. El autobús por lo general se encuentra limpio, cómodo, confortable y en buenas condiciones físicas y mecánicas para brindarme el servicio.

4. Se cuenta con las unidades de transporte suficientes, modernas y necesarias para brindar un buen servicio.

5. Las unidades de transporte están acondicionadas para brindar el servicio a personas con capacidades diferentes, de edad avanzada y embarazadas.

7. El servicio ofrece algún seguro que le permita viajar a su destino de forma confiable y protegido en caso de sufrir algún percance 0 accidente.

3. El chofer o conductor del autobús mostró tener una apariencia limpia, estar bien vestido y ofrecer buenos modales durante el servicio.

8. El servicio cumple con el horario de salidas y llegadas a cada una de las paradas programadas para la ruta.

6. El servicio que se otorga es correcto y sin errores en cada una de las escalas (paradas y llegadas) que realiza.

11. Se responde de forma personal a las solicitudes o quejas derivadas del servicio que se ofrece a los usuarios.

13. El servicio que se ofrece durante el turno nocturno, fines de semana y días no laborables responde a las necesidades de los usuarios.

12. El servicio que se ofrece durante el turno diurno, fines de semana y días no laborables responde a las necesidades de los usuarios.

1. En cuanto a la señalización e información de las paradas del autobús, éstas son consideradas adecuadas para orientar y facilitar su ubicación.

9. El servicio brindado incluye diversas formas de realizar el pago correspondiente al pasaje.

15. La realización de los viajes se hacen regularmente en tiempo de acuerdo a lo programado para cada ruta.

10. En caso de que la unidad de transporte se averíe durante su traslado, ésta ofrece un plan alterno para responder al servicio prometido.

Fuente: Elaboración propia, a partir de los datos procesados en el SPSS, v23 
A continuación, se presenta el gráfico de sedimentación, donde se aprecia las tres dimensiones que explican la mayor parte de la varianza total, con un $58.57 \%$ (Figura 1). Asimismo, el análisis factorial muestra el nuevo conjunto de variables, el cual resulta menor en número que las variables originales.

Figura 1. Gráfico de sedimentación de las variables

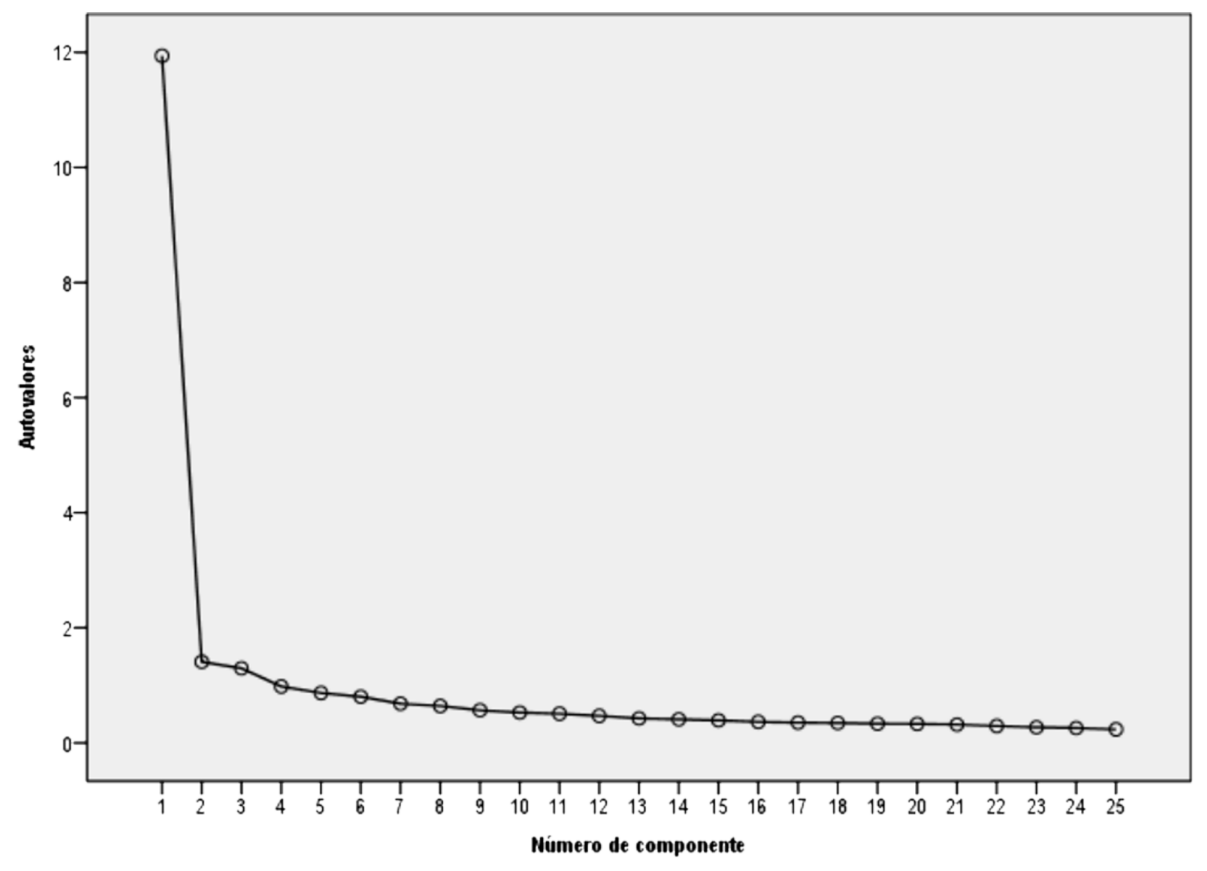

Fuente: Elaboración propia, a partir de los datos procesados en el SPSS, v23

\section{Perfil del encuestado}

La participación en la encuesta fue voluntaria; sin embargo, alcanzó al 100\% de los sujetos en condiciones de participar. La base de respuestas se vinculó con datos demográficos para la identificación y caracterización de los participantes, los cuales correspondieron a 674 hombres (52\%) y 622 mujeres (48\%). El rango de edad de los encuestados más predominante fue de 21 a 26 años.

En la Figura 2, el nivel de estudios máximo cursado más representativo son aquellos que terminaron sus estudios de preparatoria o media superior, con un $61.6 \%$, seguido por profesional, con un $22.6 \%$, en ese sentido, se destaca que 
los usuarios que más hacen uso del transporte público son los que culminaron su bachillerato y que posiblemente estén estudiando una carrera universitaria.

Figura 2. Nivel máximo de estudios del encuestado

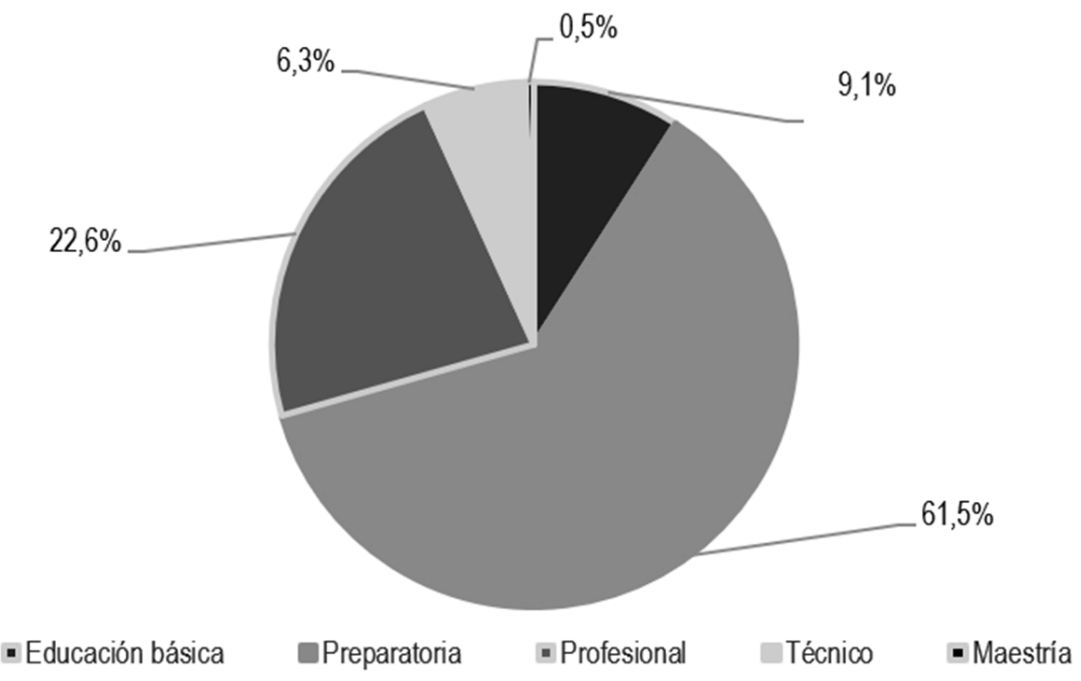

Fuente: Elaboración propia, a partir de los datos procesados en el SPSS, v23

De igual forma, se puede corroborar que la ocupación actual del encuestado es de estudiante, con un $66.2 \%$, seguido por la de empleado, con un $21.4 \%$. En cuanto al nivel de ingresos mensuales (rango), se puede observar que el rango que más predomina es el de menos de $\$ 3,000.00$ pesos mensuales.

\section{El sistema de transporte público}

Las rutas más utilizadas por los usuarios de acuerdo a la muestra seleccionada son: Línea 4-Centro; Línea 11-Luis Orci y la Línea 12. El tiempo aproximado en minutos para abordar una unidad de transporte, es aproximadamente de 11 a 25 minutos. La frecuencia de uso por parte del usuario se muestra en la Tabla 5, donde se desataca que al menos el transporte es utilizado en un par de veces al día, mientras que un $30.4 \%$ manifestó una frecuencia de uso de al menos de 3 a 5 veces al día. 
Tabla 5. Frecuencia de uso del transporte público municipal

\begin{tabular}{ccc}
\hline Rango de uso & Frecuencia & Porcentaje \\
\hline 1-2 veces por día & 689 & 53.2 \\
3-5 veces por día & 394 & 30.4 \\
Más de 5 veces por día & 13 & 1.0 \\
1-2 veces por semana & 200 & 15.4 \\
Total & 1296 & 100.0 \\
\hline
\end{tabular}

Fuente: Elaboración propia, a partir de los datos procesados en el SPSS, v23

La satisfacción del usuario está representada por la evaluación general otorgada al servicio de transporte público municipal, la cual se puede observar en la Figura 3, donde el servicio otorgado fue evaluado de pésimo a regular.

Figura 3. Evaluación otorgada al servicio de transporte público municipal

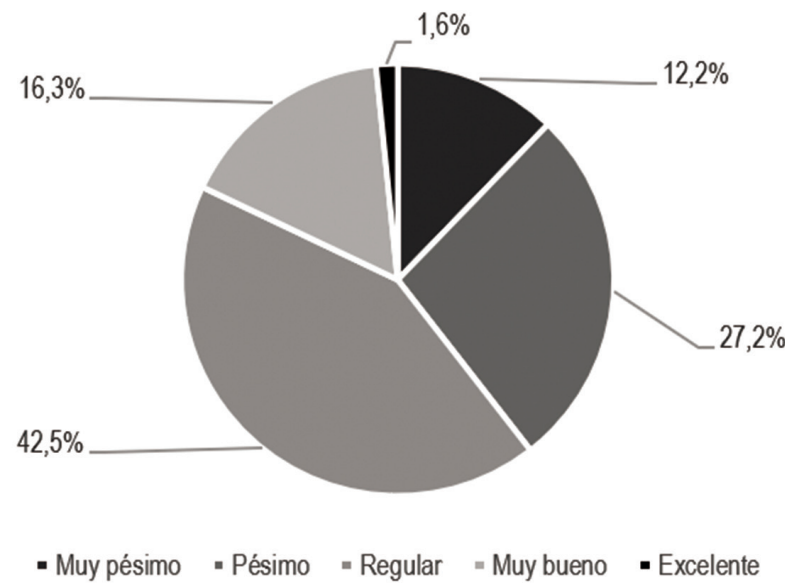

Fuente: Elaboración propia, a partir de los datos procesados en el SPSS, v23

\section{Análisis de regresión lineal múltiple}

Las variables independientes (dimensiones de la calidad en el servicio) y la variable dependiente (satisfacción del usuario) del modelo, se generaron a través de promedios ponderados con el fin de realizar el análisis de la regresión lineal múltiple.

Los estadísticos descriptivos (medias y desviaciones estándar), para las variables independientes y dependiente resultaron positivos (Tabla 6). 
Tabla 6. Medias y desviaciones estándar para las variables

\begin{tabular}{cccc}
\hline Variable & Media & Desviación típica & $\mathbf{N}^{*}$ \\
\hline Y = Satisfacción de usuario & 2.680 & .9420 & 1293 \\
X1 & 3.231 & 1.01465115 & 1293 \\
X2 & 3.261 & .91797448 & 1293 \\
X3 & 3.027 & 1.03155233 & 1293 \\
X4 & 3.201 & 1.03659177 & 1293 \\
X5 & 3.252 & .99070741 & 1293 \\
\hline
\end{tabular}

*3 valores perdidos.

Fuente: Elaboración propia, a partir de los datos procesados en el SPSS, v23

La normalidad de los datos entre "Y" y la combinación de valores de las variables independientes $\left(X_{1}, X_{2}, X_{3}, X_{4}, X_{5}\right)$, está normalmente distribuida, tal y como se aprecia en la Figura 4.

Figura 4. Histograma y normalidad de los datos.

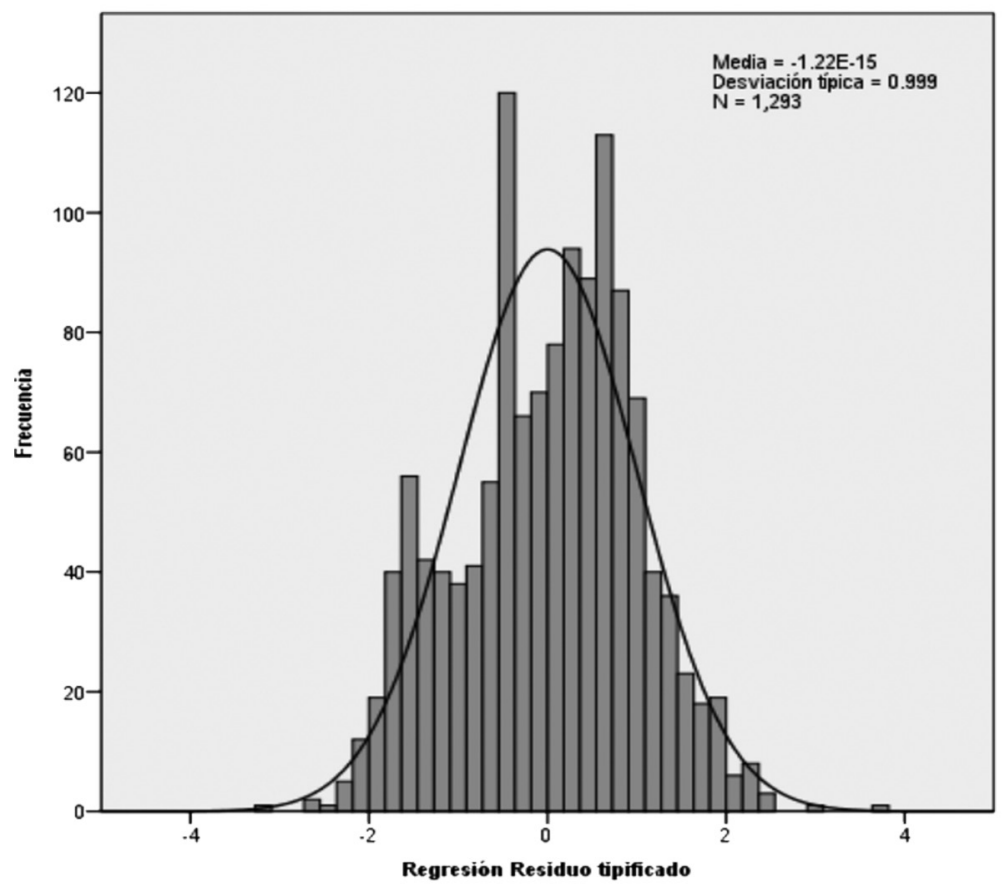

Fuente: Elaboración propia, a partir de los datos procesados en el SPSS, v23 


\section{Modelo de regresión lineal múltiple}

En la Tabla 7, se muestra el resultado de modelo generado después de aplicar el método de pasos sucesivos como opción de regresión lineal múltiple en el software estadístico SPSS v.23, en donde se detalla el coeficiente de correlación lineal de Pearson $(R)$ y el de determinación $\left(R^{2}\right)$, así como los estadísticos de cambio y el test de Durbin-Watson.

Tabla 7. Modelo de regresión lineal múltiple

\begin{tabular}{cccccccccc}
\hline $\mathbf{R}$ & $\mathbf{R}^{2}$ & $\begin{array}{c}\mathbf{R}^{2} \\
\text { ajustado }\end{array}$ & $\begin{array}{c}\text { Error } \\
\text { estándar de } \\
\text { la estimación }\end{array}$ & $\begin{array}{c}\text { Cambio } \\
\text { en } \mathbf{R}^{2}\end{array}$ & $\begin{array}{c}\text { Cambio } \\
\text { en } \mathbf{F}\end{array}$ & gl1 & gl2 & $\begin{array}{c}\text { Sig. } \\
\text { Cambio } \\
\text { en } \mathbf{F}\end{array}$ & $\begin{array}{c}\text { Estadístico } \\
\text { de Durbin- } \\
\text { Watson }\end{array}$ \\
\hline 0.449 & 0.246 & 0.244 & 0.819 & 0.006 & 10.019 & 1 & 1289 & 0.002 & 1.293 \\
\hline
\end{tabular}

Fuente: Elaboración propia, a partir de los datos obtenidos del SPSS, v.23.

Se puede apreciar una $\mathrm{R}^{2}$ igual a 0.246 , lo que significa que la bondad del ajuste lineal del modelo está explicando un $24.4 \%$ de la variabilidad en la satisfacción del usuario, a través de las dimensiones de la calidad en el servicio.

\section{Análisis de la varianza}

En la Tabla 8, se muestra el resumen del ANOVA del modelo de regresión lineal múltiple, donde el estadístico $\mathrm{F}$ permite contrastar la hipótesis nula de que el valor poblacional de $\mathrm{R}$ es cero, lo que aprueba la existencia de una relación lineal entre la variable dependiente y las dimensiones o variables independientes significativas.

El valor del nivel crítico Sig. $=0.000$, indica que las variables están linealmente relacionadas, es decir, el modelo de regresión es estadísticamente significativo, lo que indica que la variabilidad observada no se debe al azar.

Se puede asumir que existe cierta asociación entre las dimensiones que miden la calidad en el servicio y la satisfacción general del usuario. 
Tabla 8. Resumen del ANOVA del modelo de regresión lineal múltiple

\begin{tabular}{cccccc}
\hline & $\begin{array}{c}\text { Suma } \\
\text { de cuadrados }\end{array}$ & gl & $\begin{array}{c}\text { Media } \\
\text { cuadrática }\end{array}$ & F & Sig. \\
\hline Regresión & 281.520 & 3 & 93.840 & 139.954 & .000 \\
Residuo & 864.282 & 1289 & 0.671 & & \\
Total & 1145.802 & 1292 & & & \\
\hline
\end{tabular}

Predictores: (Constante), Tangibles, Seguridad, Empatía

Fuente: Elaboración propia, a partir de los datos obtenidos del SPSS, v.23.

\section{t-student}

Para conocer la significancia de las dimensiones en relación con la satisfacción del usuario, se analizaron las puntuaciones en $t$-student para cada uno de los coeficientes del total de las dimensiones. Los resultados demostraron que sólo 3 de las 5 dimensiones aportan significativamente al modelo generado. Por lo tanto, los resultados significativos fueron para las variables: Tangibles (DIM1), Seguridad (DIM4) y Empatía (DIM5), incluyendo la constante de regresión, ya que el valor del nivel crítico fue Sig.<0.05 (Tabla 9).

Tabla 9. Coeficientes de regresión para las dimensiones introducidas

\begin{tabular}{ccccccccc}
\hline Modelo & \multicolumn{2}{c}{$\begin{array}{c}\text { Coeficientes no } \\
\text { estandarizados }\end{array}$} & $\begin{array}{c}\text { Coeficiente } \\
\text { Estanda- } \\
\text { rizado }\end{array}$ & $\mathbf{t}$ & Sig. & \multicolumn{2}{c}{$\begin{array}{c}\text { Estadísticas } \\
\text { de colinealidad }\end{array}$} \\
& $\boldsymbol{\beta}$ & $\begin{array}{c}\text { Error } \\
\text { Estándar }\end{array}$ & $\boldsymbol{\beta}$ & & & Tolerancia & VIF \\
\hline (Constante) & 0.994 & 0.085 & & 11.642 & 0.000 & & \\
Tangibles & 0.219 & 0.031 & 0.235 & 7.164 & 0.000 & 0.542 & 1.846 \\
Seguridad & 0.111 & 0.035 & 0.122 & 3.165 & 0.002 & 0.392 & 2.550 \\
Empatía & 0.192 & 0.038 & 0.202 & 5.109 & 0.000 & 0.376 & 2.663 \\
\hline
\end{tabular}

Variable dependiente: Satisfacción del usuario

Fuente: Elaboración propia, a partir de los datos obtenidos del SPSS, v.23.

Para las dimensiones: Confiabilidad (DIM2) y Responsabilidad (DIM3), no fueron significativos, ya que el valor del nivel crítico fue Sig. $>0.05$.

Con respecto a los coeficientes no estandarizados del modelo de regresión, éste contiene los factores que definen la ecuación de regresión en puntuaciones directas. El coeficiente que corresponde a la Constante, es la que se ha 
denominado $\beta_{0}$, mientras que los coeficientes para Tangibles (DIM1), Seguridad (DIM4) y Empatía (DIM5), son $\beta_{1}, \beta_{4}$ y $\beta 5$, respectivamente.

A su vez, los coeficientes de regresión estandarizados y/o tipificados Beta, permiten valorar la importancia relativa de cada dimensión en la ecuación de regresión lineal múltiple. En la misma Tabla 9, se puede analizar que la dimensión significativa con mayor importancia relativa es la definida como DIM1: Tangibles, con un valor absoluto de 0.235. Una segunda dimensión fue Empatía (DIM1), con un coeficiente de 0.202 y, por último, la dimensión de Seguridad (DIM4) con un coeficiente de 0.122 .

\section{Estadísticos de multicolinealidad}

En la misma Tabla 9, se analizan los estadísticos de colinealidad, los cuales indicaron que no existen problemas de multicolinealidad entre las dimensiones o variables independientes DIM1, DIM4 y DIM5, ya que los valores del factor de inflación de la varianza (FIV) son menores a 10; es decir, resultaron valores entre 1.846 y 2.663 , mientras que todos los valores de Tolerancia $(T)$ fueron mayores a 0.10 .

Lo anterior, se refuerza con lo mostrado en la Tabla 10, donde el índice de condición de multicolinealidad generados para las dimensiones significativas resultó con valores menores a 30 , lo que indica que no existen problemas de multicolinealidad entre las dimensiones o variables independientes DIM1, DIM2 y DIM5.

Tabla 10. Diagnóstico de colinealidad

\begin{tabular}{ccccccc}
\hline \multirow{2}{*}{ Dimensión } & Autovalor & $\begin{array}{c}\text { Índice de } \\
\text { condición }\end{array}$ & \multicolumn{4}{c}{ Proporciones de varianza } \\
& & (Constante) & Tangibles & Seguridad & Empatía \\
\hline 1 & 3.887 & 1.000 & 0.00 & 0.00 & 0.00 & 0.00 \\
2 & 0.055 & 8.413 & 0.95 & 0.05 & 0.02 & 0.11 \\
3 & 0.036 & 10.337 & 0.04 & 0.07 & 0.96 & 0.19 \\
4 & 0.021 & 13.510 & 0.01 & 0.89 & 0.02 & 0.69 \\
\hline
\end{tabular}

Fuente: Elaboración propia, a partir de los datos obtenidos del SPSS, v.23. 
De acuerdo a los coeficientes del modelo de regresión lineal múltiple que se obtuvo, fue posible proponer la ecuación de regresión, tal y como se muestra en la ecuaciones 1 y 2.

$$
\begin{gathered}
Y=\beta_{0}+\beta_{1 \mathrm{DIM} 1}+\beta_{4 \mathrm{DIM} 4}+\beta_{5 \mathrm{DIM} 5}+\varepsilon \\
Y=0.994+0.219(\mathrm{DIM} 1)+0.111(\mathrm{DIM} 4)+0.192(\mathrm{DIM} 5)
\end{gathered}
$$

donde:

$\mathrm{Y}=$ Satisfacción del usuario del transporte público

$\mathrm{B}_{0}=$ Constante del modelo

$\beta_{1}, \beta_{4}, \beta_{5}=$ Betas del modelo

DIM1 = Tangibles

DIM4 = Seguridad

DIM5 = Empatía

$\varepsilon=$ Error 0 residual

Por último, los resultados estadísticos obtenidos (t-valor y sig.) de todas las dimensiones o variables independientes en relación con la satisfacción del usuario o variable dependiente, indican que sólo las hipótesis $\mathrm{H}_{1}, \mathrm{H}_{4}$ y $\mathrm{H}_{5}$ son aceptadas para el presente estudio.

\section{Conclusiones}

Actualmente, para el sector de transporte público municipal es considerable y trascendente que tenga muy bien identificadas aquellas áreas de oportunidad y mejora en cuanto a ofrecer un servicio de calidad y con ello, garantizarle al usuario la satisfacción del propio servicio.

El reconocer las dimensiones que influyen en la calidad que los clientes perciben cuando reciben un servicio, puede generar una ventaja clara para satisfacer a un usuario cada vez más preparado y con niveles de exigencia mayores.

Es importante visualizar que los coeficientes de todas las dimensiones 0 variables que fueron introducidas al modelo y que resultaron significativas, inciden en la satisfacción global del servicio de forma positiva. 
Los aspectos de tangibilidad, seguridad y empatía al momento de ofrecer y otorgar el servicio de transporte público en la ciudad de Hermosillo, parecen ser los máximos determinantes para determinar la satisfacción del usuario.

En cuanto al objetivo del presente estudio, éste se cumplió, ya que midió la calidad en el servicio mediante las cinco dimensiones propuestas por Cronin y Taylor a través de la metodología del ServPerf, resultando las dimensiones Tangibles, Seguridad y Empatía las que tuvieron un peso relativo positivo más significativo, con lo cual se responde la pregunta de investigación planteada.

En ese sentido, uno de los aportes de la investigación es que el transporte público municipal debe de poner mayor énfasis en la mejora de las unidades de trasporte en cuanto a limpieza, comodidad, confortabilidad, mejora de las condiciones físicas y mecánicas para brindar un buen servicio, resultado del modelo de regresión lineal múltiple y confirmado por el análisis factorial realizado.

De igual forma, el aspecto de seguridad es importante en cuanto al manejo eficiente de las habilidades y destrezas en la conducción por parte del chofer, así como el respeto siempre a las reglas y señalamientos de tránsito, de tal manera que brinde un nivel de confianza en el usuario de que su viaje será cómodo, seguro y que llegará a tiempo a su destino.

La empatía reveló un grado de importancia significativo, la cual fue medida a través de la atención personalizada que se brindó por parte del chofer de la unidad antes, durante y después del servicio, lo que muestra el interés por solucionar cualquier dificultad que presente durante el traslado.

Con base a lo anterior y a la ecuación de regresión generada, se puede inferir que con un $21.9 \%$ impactan los elementos tangibles en la satisfacción de los usuarios, mientras que con un $19.5 \%$, impacta la empatía y, con un $11.1 \%$, aspectos de seguridad.

Los resultados obtenidos corroboraron las hipótesis que se formularon para este estudio de investigación, la cual no se contrapone con lo encontrado en la diversa literatura y estudios empíricos ya realizados (Sánchez y Romero, 2010; Heredia, 2015).

La escala utilizada del modelo ServPerf, permitió medir la calidad percibida por los usuarios del servicio de transporte público municipal, asimismo, permitió a través del análisis estadístico, revelar qué es lo más importante desde la perspectiva de los usuarios y con ello, poder identificar aquellas áreas de oportunidad para el sistema rector del transporte, con el fin de que se gestionen y apliquen las medidas pertinentes para su mejora. 
Por último, es importante no dejar pasar por alto el resto de las dimensiones o variables independientes que fueron excluidas por el proceso estadístico, ya que cada una de ellas podría aportar al modelo y, por ende, a la satisfacción global de la calidad en el servicio. Como otra recomendación, el presente estudio se pude replicar en otras ciudades no solamente del estado, sino del país y también a nivel internacional, para determinar el impacto de cada variable y hacer un estudio comparativo que permita dimensionar los aspectos relevantes de cada una de ellas en los diferentes contextos.

\section{Referencias}

Barry, J. (2004). Urban Public Transport today; Comfort, speed, cleanliness, efficiency. USA: E. \& F. Spon.

Ben-Akiva, M., and Lerman, S. (1985). Discrete choice analysis: theory and applications to travel demand, MIT Press, Massachusetts.

Bhardwaj, R. (2011). Perception about the attributes of selected fast food retailers and their impact on consumer satisfaction and sales. Management Convergence, 1(2), 83-102.

Carman, J.M. (1990). Consumer perception of service quality: an assessment of the SERV QUAL dimensions. Journal of Retailing, 65, 33-55.

Carmines, E., and Zeller, R. (1979). Reliability and validity assessment. California: Sage.

Cronin, J.J., and Taylor, S. A. (1992). Measuring service quality: a reexamination and extension. Journal of Marketing, 56, 55-68.

(1994). SERVPERF versus SERVQUAL: Reconciling performance based and perceptions minus expectations measurement of service quality. Journal of Marketing, 58, 125-131.

Delgado Martínez, H., Prado, V.H., y Ochoa, V.H. (2011). Estudio de la calidad del servicio de transporte Urbano en Cd. Guzmán, Jalisco (desde la perspectiva del usuario). Administración de la calidad, 53-67.

Denton, K.D. (1991). Calidad en el servicio a los clientes. Edición Díaz de Santos, S. A. Madrid.

Disney, J. (1998). Competing through quality in transport services. Journal. Managing Service Quality, 8(2), 112-118.

Embretson, S., and Reise, S. (2000). Item Response Theory for psychologists. Nueva Jersey: Lawrence Erlbaum.

Frometa, G., Zayas, A.M., y Pérez, A. (2008). La Gestión de la calidad en los servicios. Contribuciones a las Ciencias Sociales, 1.

George, D., and Mallery, P. (2003). SPSS for Windows step by step: a simple guide and reference. 11.0 Update ( $4^{a} \mathrm{Ed}$.). Boston: Allyn \& Bacon. 
Grönroos, C. (1994). Marketing y gestión de servicios: la gestión de los momentos de la verdad y la competencia en los servicios. Madrid: Editorial Díaz de Santos.

Grönroos, C. (2001). The perceived service quality concept - a mistake? Managing service quality, 11(3), 150-152.

Hattie, J. (1985). Methodology review: assessing unidimensionality of tests and items. Applied Psychological Measurement, 9(2), 139-164.

Heredia, J.K. (2015). Modelo de satisfacción de los usuarios de transporte público tipo bus integrando variables latentes. Universidad Nacional de Colombia. Tesis inédita para obtener el grado de Magister en Ingeniería-Infraestructura y Sistemas de Transporte. Medellín, Colombia.

Hoffman, D., y Bateson, J. (2012). Fundamentos de marketing de servicios. México. Cengage Learning, 324.

Hojman, P., Ortúzar, J. de D., y Rizzi, L. (2003). El valor de la reducción de accidentes fatales y no fatales graves en carretera. Actas XI Congreso Chileno de Ingeniería del Transporte, Santiago, Chile, 515-525.

Hovell, P. (1999). Some Organizational Problems Facing Urban Passenger Transport. USA. Juran, J. y Gryna, F. (1993). Manual de Control de Calidad. (s.l.): Madrid: McGraw-Hill.

Khan, S., Majid, S., and Yaqoob, F. (2013). Determinants of Customer Satisfaction in Fast Food Industry. A Study of Fast Food Restaurants Peshawar Pakistan. Studia commercialia Bratislavensia, 6(21), 56-65.

Kotler, P., y Armstrong, G. (2013). Fundamentos de Marketing. México. Pearson Educación. Lovelock, Ch., Huete, L., y Reynoso, J. (2010). Administración de servicios: Estrategias para la creación de valor en el nuevo paradigma de los negocios. U.S.A. Addison-Wes ley.

Lovelock, C.H. (1990). Services Marketing. Series in Marketing. New Jersey: Prentice Hall. Merino, C., Navarro, J., and García, W. (2014). Revisión de la consistencia interna del inventario de inteligencia emocional de Bar-On, EQ-I: YV. Revista Peruana de Psicología y Trabajo Social, 3(1), 141-154.

Molinero, Á., y Sánchez, L.I. (1997). Transporte Público Planeación, Diseño, Operación y Administración. Universidad Autónoma del Estado de México, México.

Morillo, M., y Morillo, M. Del C. (2016). Satisfacción del usuario y calidad del servicio en alojamientos turísticos del estado Mérida, Venezuela. Revista de Ciencias Sociales, 12(2).

Münch, L. (1998). Más allá de la excelencia y de la calidad total. México. Editorial Trillas.

Navarrete Rodríguez, P. E., y Andrade, M.A. (2010). La infraestructura del transporte público urbano en la Ciudad de México y su relación con las políticas públicas. Investigación Administrativa, 41-59.

Ortúzar, J. de D. (2000). Modelling route and multimodal choices with revealed and stated preferences data, en J. de D. Ortúzar (ed.), Stated Preference Modelling Techniques, Perpectives 4, PTRC Education and Research Services, Londres. 
Parasuraman, A., Zeithaml, V., and Berry, L. (1985). Conceptual model of service quality and its implications for future research. Journal of marketing, 49, 41-50.

Parasuraman, A., Zeithaml, V., and Berry, L. (1988). SERVQUAL: a multiple-item scale for measuring consumer perceptions of services quality. Journal of Retailing, 64(1), 12-40.

Peel, M. (1993). El servicio al cliente: guía para mejorar la atención y la asistencia. España: Ediciones Deusto.

Phau, I., and Ferguson, G. (2013). Validating the customer satisfaction survey (CSS) Scale in the Australian fast food industry. Australasian Marketing Journal, 21, 147-154.

Rizzi, L., and Ortúzar, J. de D. (2003). Stated preference in the valuation of interurban road safety. Accident Analysis and Prevention, 35, Oxford, 9-22.

Sánchez-Flores, Óscar y Romero-Torres, Javier. (2010). Factores de calidad del servicio en el transporte público de pasajeros: estudio de caso de la ciudad de Toluca, México. Economía, sociedad y territorio, 10(32), 49-80.

Sancho, A. (1998). Introducción al Turismo. Madrid. Organización Mundial del Turismo.

Sussman, J. (2000). Introduction to Transportation Systems, Artech House, Boston.

Vásquez, R. (2010). La satisfacción del cliente, nuevos retos para la gestión de calidad en el sector hotelero, en el cambio de época, en http://www.monografias.com/trabajos 82/satisfaccion-cliente-sector-hotelero/satisfaccion-cliente-sector-hotelero2.shtml

Zeithaml, V.A. (1988). Consumer Perceptions of Price, Quality and Value: A Means-End Model and Synthesis of Evidence. Journal of Marketing, 52, 2-22.

Zeithaml, V., and Parasuraman, A. (2004). Relevant knowledge series: Service Quality. Cambridge, Mass.: Marketing Science Institute. 